\title{
Probing the origin of elliptical high-order harmonic generation from aligned molecules in linearly polarized laser fields
}

\author{
Sang-Kil Son (손상길), ${ }^{1, *}$ Dmitry A. Telnov, ${ }^{2, \dagger}$ and Shih-I Chu (朱時宜) ${ }^{1,3, \dagger}$ \\ ${ }^{1}$ Department of Chemistry, University of Kansas, Lawrence, Kansas 66045, USA \\ ${ }^{2}$ Department of Physics, St. Petersburg State University, St. Petersburg 198504, Russia \\ ${ }^{3}$ Center for Quantum Science and Engineering, Department of Physics, National Taiwan University, Taipei 10617, Taiwan
}

(Received 26 March 2010; published 18 October 2010)

\begin{abstract}
A recent experiment [Phys. Rev. Lett. 102, 073902 (2009)] has demonstrated that elliptically polarized highorder harmonic generation can be produced from linearly polarized driving fields for aligned molecular systems. In order to reveal the underlying physical mechanisms of elliptical harmonics, we present fully $a b$ initio and high-precision calculations and analyses of the amplitude, phase, and polarization state of the harmonic radiation from molecular hydrogen ions with arbitrary orientation. We find that high ellipticity arises from molecular orbital symmetry and two-center interference effects. Our $a b$ initio exploration and findings lead to a general rule that the ellipticity becomes high for molecular orbitals represented by a symmetric combination of atomic orbitals, whereas it becomes low for molecular orbitals represented by an antisymmetric combination. This finding also applies to the general case of aligned linear molecules.
\end{abstract}

DOI: 10.1103/PhysRevA.82.043829

PACS number(s): 42.65.Ky, 31.15.A-

High-order harmonic generation (HHG) is one of the most rapidly developing topics in strong-field atomic, molecular, and optical physics today, leading to the recent advancement of the attosecond science [1-3] and the feasibility of frequency-comb generation in the extreme ultraviolet to vacuum ultraviolet regimes [4-7], etc. Traditionally, the power (or radiation energy) spectra have been the main topic of HHG studies both experimentally and theoretically. However, recent developments in molecular alignment technology [8] open a possibility of investigating other HHG properties including the phase [9-11] and polarization [12-14] of the harmonic radiation.

For ensembles of atoms or unaligned molecules, the polarization of the harmonics is expected to be the same as the polarization of the driving laser field. For aligned molecules, however, the harmonic radiation has two components: one parallel and another perpendicular to the laser polarization direction. Experiments [12,13] showed that the linear polarization state of HHG driven by linearly polarized laser fields is tilted due to the nonvanishing perpendicular component of HHG. Recently, the elliptical polarization state of HHG driven by linearly polarized laser fields has been measured [14]; speculations on its possible origins include, for example, multielectron effects and the influence of the Coulomb potential. An experiment [14] showed high ellipticity for $\mathrm{N}_{2} \mathrm{HHG}$ but no ellipticity for $\mathrm{CO}_{2} \mathrm{HHG}$. Controversially, a theory including multiple electronic continuum dynamics predicts elliptical $\mathrm{HHG}$ from aligned $\mathrm{CO}_{2}$ [15]. In general, according to the selection rules [16], elliptical harmonic radiation is expected unless the molecular alignment is parallel or perpendicular to the driving laser polarization. The widely used strong-field approximation (SFA), however, cannot predict this ellipticity of HHG at all because only the parallel component

\footnotetext{
*sangkil.son@cfel.de; Present address: Center for Free-Electron Laser Science, DESY, 22607 Hamburg, Germany.

†telnov@pcqnt1.phys.spbu.ru

${ }^{\ddagger}$ sichu@ku.edu
}

of the electronic momentum is considered in the SFA. A few modifications of the SFA have been proposed, but the perpendicular component of the harmonic radiation predicted is negligibly small [17] and so is the computed ellipticity [18]. Since the mechanism of the elliptical HHG is still under debate and largely unknown, it is most desirable and timely to perform a fully $a b$ initio study to explore and clarify the origin of the ellipticity.

In this article, we present fully $a b$ initio and high-precision calculations of the time-dependent Schrödinger equation in three dimensions to explore the origin of elliptical HHG from the ground and excited electronic states of $\mathrm{H}_{2}{ }^{+}$with arbitrary orientation. In our $a b$ initio calculations, the influence of the Coulomb potential is fully incorporated and there are no multielectron effects for the one-electron system. For a high-precision solution of the wave function in space and time, we use the time-dependent generalized pseudospectral method (TDGPS) [19]. The electronic structure of the two-center system is accurately solved on nonuniform and optimal grids in prolate spheroidal coordinates [20], and the wave function is efficiently and accurately propagated in time using the split-operator technique in the energy representation [21]. Recently, the TDGPS method has been applied successfully to investigate the orientation effects in multiphoton ionization and $\mathrm{HHG}$ of $\mathrm{H}_{2}{ }^{+}$[19] and other diatomic molecules [22,23]. Detailed numerical procedures can be found in Ref. [19].

Figure 1(a) displays alignment of a linear molecule and the components of the induced dipole moment parallel and perpendicular to the laser polarization direction. We assume perfect molecular alignment. Without loss of generality, we assume that the linear molecule is aligned along the $z$ axis and the polarization vector of the driving laser field lies in the $x z$ plane with orientation angle $\Theta$. The $y$ axis is chosen as the propagation direction of the driving laser field. Then, the induced time-dependent dipole moment $\mathbf{d}(t)$ has two components with respect to the molecular axis: $d_{x}(t)=\langle\psi(t)|x| \psi(t)\rangle$ and $d_{z}(t)=\langle\psi(t)|z| \psi(t)\rangle$, where $\psi(t)$ is the wave function propagated in time. The function $d_{y}(t)$ is zero for this alignment. The vector quantity $\mathbf{d}(t)$ can be 


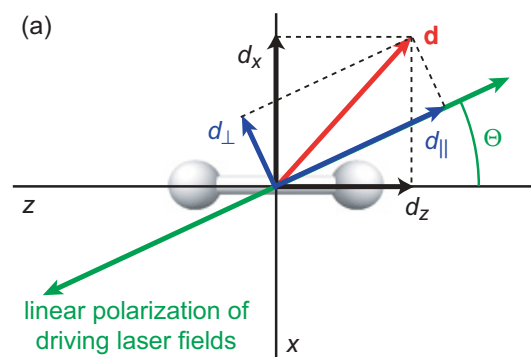

(b)

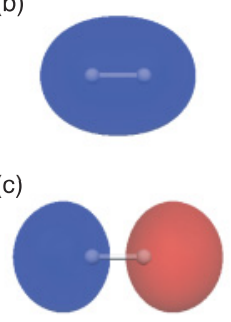

FIG. 1. (Color online) (a) A linear molecule is aligned to the $z$ axis, and the driving laser field is linearly polarized with the orientation angle $\Theta$. The induced dipole moment producing the harmonic radiation has two components $d_{z}$ and $d_{x}$ with respect to the molecular axis. The same vector is represented by $d_{\|}$and $d_{\perp}$, which are parallel and perpendicular to the laser polarization direction, respectively. Panels (b) and (c) show pictures of the symmetric $1 \sigma_{g}$ orbital and the antisymmetric $1 \sigma_{u}$ orbital of $\mathrm{H}_{2}{ }^{+}$, respectively.

explicitly represented by the parallel $(\|)$ and perpendicular $(\perp)$ components with respect to the laser polarization direction:

$$
\begin{aligned}
& d_{\|}(t)=d_{x}(t) \sin \Theta+d_{z}(t) \cos \Theta, \\
& d_{\perp}(t)=d_{x}(t) \cos \Theta-d_{z}(t) \sin \Theta .
\end{aligned}
$$

Now the harmonic radiation generated by the induced dipole moment has two components (parallel and perpendicular) and each component has its amplitude and phase. To compute them, we use the Fourier transform of the dipole moments with the radiation frequency $\omega ; \tilde{d}_{\|, \perp}(\omega)=$ $\int_{-\infty}^{\infty} d_{\|, \perp}(t) e^{i \omega t} d t$, which is a complex number. The phase of the harmonic radiation is given by $\phi_{\|, \perp}(\omega)=\arg \left[\tilde{d}_{\|, \perp}(\omega)\right]$ and its amplitude is $A_{\|, \perp}(\omega)=\sqrt{4 \omega^{4} /\left(6 \pi c^{3}\right)}\left|\tilde{d}_{\|, \perp}(\omega)\right|$, where $c$ is the speed of light. The spectral density of the radiation energy emitted over all time is then given by

$$
S(\omega)=\frac{4 \omega^{4}}{6 \pi c^{3}}\left|\int_{-\infty}^{\infty} \mathbf{d}(t) e^{i \omega t} d t\right|^{2}=\left[A_{\|}(\omega)\right]^{2}+\left[A_{\perp}(\omega)\right]^{2} .
$$

The ellipticity $\varepsilon$ is determined by the amplitude ratio and the phase difference of the two components:

$$
\varepsilon=\sqrt{\frac{1+r^{2}-\sqrt{1+2 r^{2} \cos 2 \delta+r^{4}}}{1+r^{2}+\sqrt{1+2 r^{2} \cos 2 \delta+r^{4}}}},
$$

where $r=A_{\perp} / A_{\|}$and $\delta=\phi_{\perp}-\phi_{\|}$. The range of the ellipticity is $0 \leqslant \varepsilon \leqslant 1$. If one of the two components has zero amplitude or the two components are in phase $(\delta=0)$, then $\varepsilon=0$ and the radiation is completely linearly polarized. If the two components have the same amplitude and are $90^{\circ}$ out of phase, then $\varepsilon=1$ and the radiation is circularly polarized. In other words, the ellipticity becomes low if the amplitudes of the two components are very different or if their phase difference is close to zero. In order to attain high ellipticity, both of the following conditions must be satisfied [15]: (i) the amplitudes of the two components are of the same order and (ii) their phase difference is around $\pm \pi / 2$.

Figures 2(a) to 2(c) present harmonic signals of the 57th harmonic from the ground state $\left(1 \sigma_{g}\right)$ of $\mathrm{H}_{2}{ }^{+}$at the laser peak

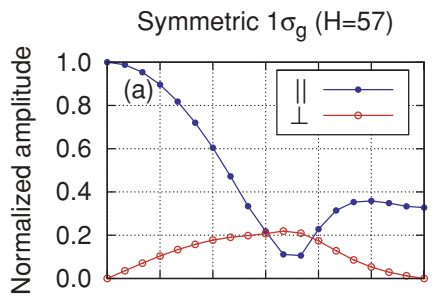

Antisymmetric $1 \sigma_{\mathrm{u}}(\mathrm{H}=31)$
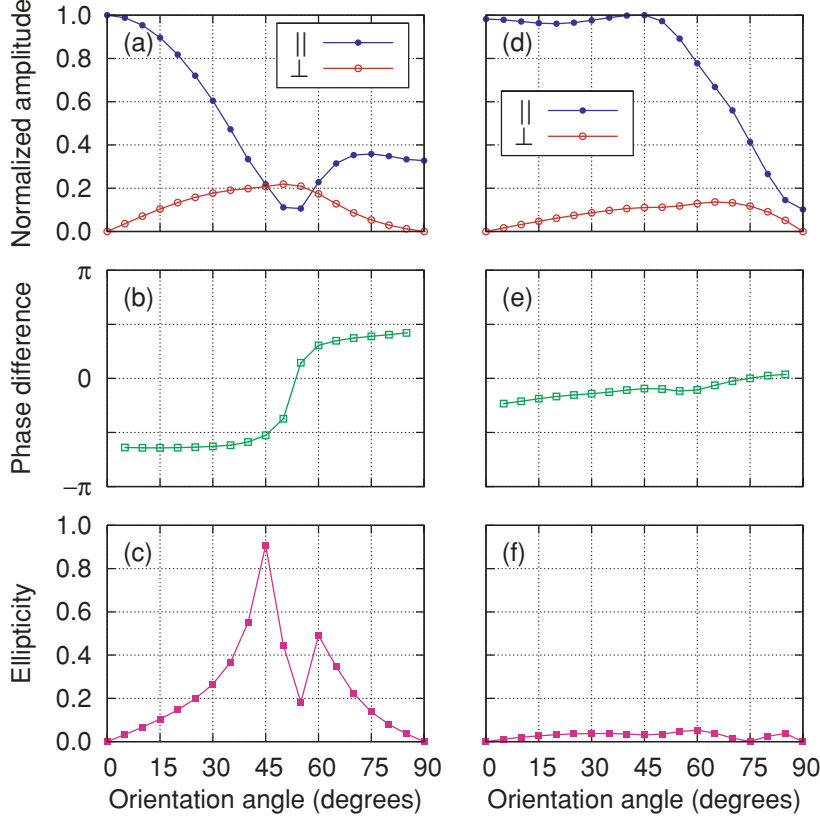

FIG. 2. (Color online) Amplitude, phase, and ellipticity of harmonic signals as a function of orientation angle: Panels (a)-(c) show the 57th harmonic $(H=57)$ from the $1 \sigma_{g}$ state of $\mathrm{H}_{2}{ }^{+}$at the intensity $3 \times 10^{14} \mathrm{~W} / \mathrm{cm}^{2}$, and panels (d)-(f) show the $31 \mathrm{st}$ harmonic $(H=31)$ from the $1 \sigma_{u}$ state of $\mathrm{H}_{2}{ }^{+}$at the intensity $2 \times 10^{14} \mathrm{~W} / \mathrm{cm}^{2}$. Panels (a) and (d) show the normalized amplitudes of the parallel $(\|)$ and perpendicular $(\perp)$ components, panels (b) and (e) show their phase difference, and panels (c) and (f) show the computed ellipticity.

intensity of $3 \times 10^{14} \mathrm{~W} / \mathrm{cm}^{2}$ and Figs. 2(d) to 2(f) present the 31 st harmonic from the first excited state $\left(1 \sigma_{u}\right)$ of $\mathrm{H}_{2}{ }^{+}$ at $2 \times 10^{14} \mathrm{~W} / \mathrm{cm}^{2}$ as a function of the orientation angle. We use the wavelength of $800 \mathrm{~nm}$ and the sine-squared pulse shape with the 20-optical-cycle pulse length. The upper panels [Figs. 2(a) and 2(d)] show the amplitudes of the parallel $\left(A_{\|}\right)$and perpendicular $\left(A_{\perp}\right)$ components of the harmonic signal normalized to the maximum value. The middle panels [Figs. 2(b) and 2(e)] indicate the phase difference between the two components. The bottom panels [Figs. 2(c) and 2(f)] show the ellipticity computed from the amplitudes and phases. Our results indicate that $r, \delta$, and $\varepsilon$ in the plateau region of the HHG spectra are nearly independent of the laser intensity in the range used in the calculations $\left(2 \times 10^{14}\right.$ to $\left.3 \times 10^{14} \mathrm{~W} / \mathrm{cm}^{2}\right)$.

First, let us consider the parallel component. The orientation dependence of the parallel component manifests an extremum which can be explained by the two-center interference effect. According to the recollision model [24,25], the harmonic signal exhibits an interference minimum or maximum depending on the phase difference of the returning electron wave between the two nuclei in the diatomic molecule. The extremum position with respect to the orientation angle is given by [25]

$$
\cos \Theta=\frac{\pi}{R \sqrt{2 N \omega_{0}}},
$$

where $N$ is the harmonic order, $\omega_{0}$ is the incident laser frequency, and $R$ is the distance between the two centers (i.e., 
the bond length for diatomic molecules). Equation (4) assumes that all the kinetic energy of the electron is converted into the harmonic radiation energy upon recollision; this dispersion relationship is well satisfied in the plateau region of the $\mathrm{HHG}$ spectrum [9]. If the wave function is represented by a symmetric combination of atomic orbitals (AOs); for example, $1 \sigma_{g}$ of $\mathrm{H}_{2}{ }^{+}$as shown in Fig. 1(b), then Eq. (4) yields destructive interference and points at the minimum, as shown by the solid circles in Fig. 2(a). On the other hand, if the wave function is represented by an antisymmetric combination of AOs like $1 \sigma_{u}$ of $\mathrm{H}_{2}{ }^{+}$in Fig. 1(c), then Eq. (4) gives constructive interference and points at the maximum, as shown by the solid circles in Fig. 2(d). The extremum positions from our calculations agree well with the angles estimated from Eq. (4) [19].

Regarding the perpendicular component, there have been only a few discussions on this topic because its amplitude is usually small in comparison with the parallel component $[17,24]$. Since there is no explicit driving field in this direction, the perpendicular component of the harmonic radiation is not subject to the simple recollision model. To obtain a semiquantitative description of its orientation dependence in our calculations, we can employ first-order perturbation theory. Since a linear molecule possesses an axial symmetry, there are two different polarizabilities $\alpha_{x}$ and $\alpha_{z}$ which relate the induced dipole moments $d_{x}$ and $d_{z}$ to the driving field:

$$
\begin{aligned}
& d_{x}(t)=\alpha_{x} E_{x}(t)=\alpha_{x} E(t) \sin \Theta, \\
& d_{z}(t)=\alpha_{z} E_{z}(t)=\alpha_{z} E(t) \cos \Theta .
\end{aligned}
$$

Then, from Eq. (1), one can obtain

$$
d_{\perp}(t)=\frac{1}{2}\left(\alpha_{x}-\alpha_{z}\right) E(t) \sin 2 \Theta .
$$

Equation (6) nicely describes the orientation-dependent pattern of the numerical results, showing a smooth convex curve approaching zero at $\Theta=0^{\circ}$ or $90^{\circ}$, regardless of the symmetry of the wave function. Note that $d_{\perp}(t)$ does not vanish if molecular polarizability is anisotropic.

For a symmetric combination of AOs, when the two-center interference effect for the parallel component is strong enough, the minimum of $A_{\|}$can be very deep with the $A_{\perp}$ value larger than $A_{\|}$. In this case, $A_{\perp}$ and $A_{\|}$cross in the vicinity of the minimum, as clearly shown in Fig. 2(a). This phenomenon has already been pointed out in a two-dimensional model calculation [24]. Note that the crossing pattern satisfies the first requirement for the high ellipticity: the two components have the same order of magnitude when they are crossing. According to the two-center interference model [24,25], the sudden phase jump by $\pi$ is accompanied by this minimum interference position. Our numerical results show that $\phi_{\|}$has the $\pi$ jump and $\phi_{\perp}$ is almost constant. Thus, the phase difference of $\phi_{\perp}-\phi_{\|}$suddenly jumps by $\pi$ passing through $+\pi / 2$ or $-\pi / 2$ when the amplitudes of the two components meet together, as shown in Fig. 2(b). Note that this fact satisfies the second requirement for high ellipticity: the phase difference is around $\pm \pi / 2$. Consequently, the harmonic radiation of $\mathrm{H}_{2}{ }^{+}$ $1 \sigma_{g}$ is expected to have a significant ellipticity, except for the parallel $\left(\Theta=0^{\circ}\right)$ and perpendicular $\left(\Theta=90^{\circ}\right)$ molecular alignment. In Fig. 2(c), the ellipticity becomes high when both conditions for the amplitude and phase are satisfied. Our results indicate that high ellipticity arises for the orientation where the (a) Symmetric $1 \sigma_{\mathrm{g}}$

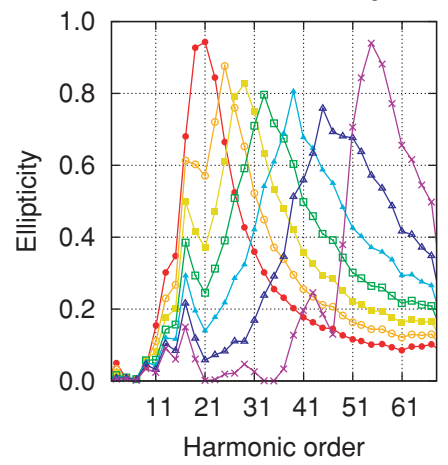

(b) Antisymmetric $1 \sigma_{\mathrm{u}}$

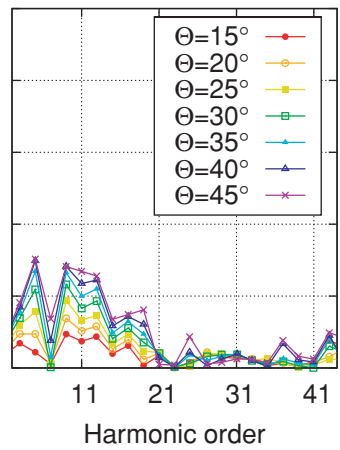

FIG. 3. (Color online) Ellipticity of the harmonic signals from $\mathrm{H}_{2}{ }^{+}$with various orientation angles: (a) the $1 \sigma_{g}$ state at the intensity $3 \times 10^{14} \mathrm{~W} / \mathrm{cm}^{2}$ and (b) the $1 \sigma_{u}$ state at the intensity $2 \times 10^{14} \mathrm{~W} / \mathrm{cm}^{2}$. The legend in (b) applies to both (a) and (b).

parallel component of the harmonic radiation has a minimum due to destructive two-center interference. According to the two-center interference model [24,25], this minimum exists in the orientation dependence of the HHG signal if the wave function is a symmetric combination of AOs. Therefore, high ellipticity can be expected for the molecular orbitals represented by a symmetric combination of AOs.

On the other hand, the situation is different for an antisymmetric combination of AOs. In Fig. 2(d), one can see that both components have a maximum, so crossing or approaching rarely occurs except at the end points. Also, our numerical results show no $\pi$ jump in the phase for both components. Consequently, the harmonic radiation of $\mathrm{H}_{2}{ }^{+} 1 \sigma_{u}$ exhibits low ellipticity, as shown in Fig. 2(f).

In Fig. 3, one can examine the harmonic-order dependence and the orientation dependence of the computed ellipticity for (a) the $1 \sigma_{g}$ state at the intensity $3 \times 10^{14} \mathrm{~W} / \mathrm{cm}^{2}$ and (b) the $1 \sigma_{u}$ state at the intensity $2 \times 10^{14} \mathrm{~W} / \mathrm{cm}^{2}$. It is clearly shown that the ellipticity of the symmetric $1 \sigma_{g}$ state is much higher than that of the antisymmetric $1 \sigma_{u}$ state. Our $a b$ initio calculations reveal that the one-electron system with no multielectron effects can generate elliptical harmonic radiation from linearly polarized laser fields if the wave function is represented by the symmetric combination of AOs. In addition, Fig. 3 shows that the ellipticity strongly depends on the orientation angle. For the $1 \sigma_{g}$ state, the harmonic order of the highest ellipticity increases as the orientation angle increases. This is well explained by Eq. (4): the harmonics of a higher order have interference minimum positions at larger orientation angles.

In summary, we have investigated the theoretical origins of the elliptical harmonic radiation from aligned linear molecules by performing high-precision ab initio calculations for $\mathrm{H}_{2}{ }^{+}$in a linearly polarized strong laser field with arbitrary orientation. Our numerical results and analyses show that all harmonic radiations have parallel and perpendicular components with respect to the driving laser polarization if the molecular orientation angle is neither 0 nor $90^{\circ}$, thus resulting in the elliptical HHG for the intermediate orientation angles. Based on the two-center interference model for the parallel 
component and the first-order perturbation approximation for the perpendicular component, we propose an instructive prediction for the ellipticity of molecular HHG related to the symmetry of the wave function: A symmetric combination of atomic orbitals produces high ellipticity, whereas an antisymmetric combination of atomic orbitals yields low ellipticity.

We can generalize this prediction for other linear molecules. For $\mathrm{H}_{2}$ with the highest-occupied molecular orbital (HOMO) approximated by a symmetric combination of atomic orbitals, the harmonic radiation is expected to be highly elliptical. For $\mathrm{O}_{2}, \mathrm{~F}_{2}$, and $\mathrm{CO}_{2}$ with their HOMO approximated by an antisymmetric combination of atomic orbitals, the harmonic radiation is expected to exhibit low ellipticity. For $\mathrm{N}_{2}$, we expect a more complicated picture because its HOMO $\left(3 \sigma_{g}\right)$ consists of both symmetric and antisymmetric combinations
[26] and undergoes a possible resonance with HOMO-1 $\left(1 \pi_{u}\right)$ in the 800-nm laser field [23]. This can be a reason for a weak orientation dependence of the HHG ellipticity in $\mathrm{N}_{2}$ as well as a striking difference between $\mathrm{N}_{2}$ and $\mathrm{CO}_{2}$ observed in a recent experiment [14]. Further investigations regarding the HHG ellipticity in multielectron diatomic and small polyatomic molecules are in progress.

This work was partially supported by the Chemical Sciences, Geosciences, and Biosciences Division of the Office of Basic Energy Sciences, Office of Sciences, U.S. Department of Energy and by the U.S. National Science Foundation. We also would like to acknowledge the partial support of National Science Council of Taiwan (Grant No. 97-2112-M-002-003MY3) and National Taiwan University (Grants No. 98R0045 and No. 99R80870).
[1] F. Krausz and M. Ivanov, Rev. Mod. Phys. 81, 163 (2009).

[2] P. B. Corkum and F. Krausz, Nature Phys. 3, 381 (2007).

[3] J. J. Carrera, X. M. Tong, and S. I. Chu, Phys. Rev. A 74, 023404 (2006).

[4] C. Gohle, T. Udem, M. Herrmann, J. Rauschenberger, R. Holzwarth, H. A. Schuessler, F. Krausz, and T. W. Hänsch, Nature (London) 436, 234 (2005).

[5] R. J. Jones, K. D. Moll, M. J. Thorpe, and J. Ye, Phys. Rev. Lett. 94, 193201 (2005).

[6] J. J. Carrera, S.-K. Son, and S. I. Chu, Phys. Rev. A 77, 031401 (2008).

[7] J. J. Carrera and S. I. Chu, Phys. Rev. A 79, 063410 (2009).

[8] H. Stapelfeldt and T. Seideman, Rev. Mod. Phys. 75, 543 (2003).

[9] X. Zhou, R. Lock, W. Li, N. Wagner, M. M. Murnane, and H. C. Kapteyn, Phys. Rev. Lett. 100, 073902 (2008).

[10] W. Boutu, S. Haessler, H. Merdji, P. Breger, G. Waters, M. Stankiewicz, L. J. Frasinski, R. Taieb, J. Caillat, A. Maquet, P. Monchicourt, B. Carre, and P. Salieres, Nature Phys. 4, 545 (2008).

[11] O. Smirnova, Y. Mairesse, S. Patchkovskii, N. Dudovich, D. Villeneuve, P. Corkum, and M. Y. Ivanov, Nature (London) 460, 972 (2009).

[12] J. Levesque, Y. Mairesse, N. Dudovich, H. Pépin, J.-C. Kieffer, P. B. Corkum, and D. M. Villeneuve, Phys. Rev. Lett. 99, 243001 (2007).
[13] G. H. Lee, I. J. Kim, S. B. Park, T. K. Kim, and C. H. Nam, Opt. Lett. 33, 2083 (2008).

[14] X. Zhou, R. Lock, N. Wagner, W. Li, H. C. Kapteyn, and M. M. Murnane, Phys. Rev. Lett. 102, 073902 (2009).

[15] O. Smirnova, S. Patchkovskii, Y. Mairesse, N. Dudovich, D. Villeneuve, P. Corkum, and M. Y. Ivanov, Phys. Rev. Lett. 102, 063601 (2009).

[16] S. Ramakrishna, P. A. J. Sherratt, A. D. Dutoi, and T. Seideman, Phys. Rev. A 81, 021802 (2010).

[17] C. C. Chirilă and M. Lein, Phys. Rev. A 80, 013405 (2009).

[18] A. Etches, C. B. Madsen, and L. B. Madsen, Phys. Rev. A 81, 013409 (2010).

[19] D. A. Telnov and S. I. Chu, Phys. Rev. A 76, 043412 (2007).

[20] X. Chu and S. I. Chu, Phys. Rev. A 63, 013414 (2000).

[21] X. M. Tong and S. I. Chu, Chem. Phys. 217, 119 (1997).

[22] D. A. Telnov and S. I. Chu, Phys. Rev. A 79, 041401 (2009).

[23] D. A. Telnov and S. I. Chu, Phys. Rev. A 80, 043412 (2009).

[24] M. Lein, N. Hay, R. Velotta, J. P. Marangos, and P. L. Knight, Phys. Rev. Lett. 88, 183903 (2002).

[25] M. Lein, N. Hay, R. Velotta, J. P. Marangos, and P. L. Knight, Phys. Rev. A 66, 023805 (2002).

[26] B. Zimmermann, M. Lein, and J. M. Rost, Phys. Rev. A 71, 033401 (2005). 